\title{
„Spielarten des Kapitalismus“ als Schule der vergleichenden Staatstätigkeitsforschung
}

\author{
Martin Höpner
}

Zusammenfassung: Der Beitrag knüpft an die von Manfred G. Schmidt entwickelte Unterscheidung von Schulen der vergleichenden Staatstätigkeitsforschung an und schlägt eine Erweiterung der unterschiedenen Schulen um die „Spielarten des Kapitalismus"-Literatur (Varieties of Capitalism, VoC) vor. Dieser Literaturzweig zielte ursprünglich auf Analyse der institutionellen Grundlagen der Wettbewerbskraft von Unternehmen und Volkswirtschaften. Wie der Beitrag im Einzelnen darlegt, haben sich die sechs Grundannahmen dieser Literatur zu einer Perspektive verdichtet, aus der systematisch distinkte Hypothesen zur vergleichenden Staatstätigkeitsforschung folgen. Dies wird anhand von drei Beispielen gezeigt: anhand der ,revisionistischen“ Wohlfahrtsstaatsdebatte, anhand neuerer Forschung zur geschlechtsspezifischen Segregation und anhand von Thesen zu den produktionsregimebezogenen Voraussetzungen erfolgreicher makroökonomischer Steuerung. Der Beitrag schließt mit einer Diskussion der Implikationen der vorgeschlagenen Erweiterung für die vergleichende Staatstätigkeitsforschung.

Schlüsselwörter: Vergleichende Staatstätigkeitsforschung · Vergleichende Politische Ökonomie $\cdot$ Spielarten des Kapitalismus

\section{"Varieties of Capitalism" as a School of Comparative Policy Research}

\begin{abstract}
This article refers to Manfred G. Schmidt's distinction between different schools of comparative policy research and proposes expanding the list of schools by including the varieties of capitalism ( $\mathrm{VoC}$ ) literature as a distinct school. Originally, VoC aimed at examining the institutional foundations of the level of competitiveness of firms and nations. As the article shows, the six basic concepts of $\mathrm{VoC}$ condensed into a perspective that allows for deduction of hypotheses in comparative policy research. The article provides three illustrations: the new "revisionist" debate on the welfare state, new research on gender-specific segregation, and theses on the production regime-oriented preconditions of successful macroeconomic policy. The article ends with a discussion about the implications for future comparative policy research.
\end{abstract}

Online publiziert: 18.09 .2009

(C) Die Autor(en) 2009. Dieser Artikel ist auf Springerlink.com mit Open Access verfügbar.

Ich danke den Teilnehmern des Institutskolloquiums des IPW der Ruprecht-Karls-Universität Heidelberg am 14. Januar 2009 sowie zwei anonymen Gutachtern der Zeitschrift für Vergleichende Politikwissenschaft für wertvolle Anregungen.

PD Dr. M. Höpner $(\bowtie)$

Max-Planck-Institut für Gesellschaftsforschung, Paulstraße 3, 50676 Köln, Deutschland

E-Mail: hoepner@mpifg.de 
Keywords: Comparative policy research · Comparative political economy ·

Varieties of capitalism

\section{Einleitung: Wie viele Schulen der vergleichenden Staatstätigkeitsforschung?}

Die von Manfred G. Schmidt entwickelte Unterscheidung von Schulen der vergleichenden Staatstätigkeitsforschung hat Einzug in die Lehrbücher gefunden und darf zum festen Kanon der vergleichenden Politikwissenschaft gerechnet werden (siehe beispielsweise Schmidt 1995, S. 576-587; Zohlnhöfer 2007, S. 157-164 sowie die Beiträge in Schmidt et al. 2007, Abschn. I). Die Leistung dieser Unterscheidung liegt vor allem darin, dass sie dem Anwender ein Grundraster an Theorieangeboten zur Verfügung stellt, dessen Bestandteile sich zu unterschiedlichen politikfeld- oder problemspezifischen Teiltheorien kombinieren lassen. In minimaler Abwandlung lassen sich mit Schmidt folgende Theorieschulen unterscheiden:

1. Theorien der sozioökonomischen Determination

2. Machtressourcen-Theorien

3. Theorien der Parteiendifferenz

4. Politisch-institutionalistische Theorien

5. Theorien der Internationalisierung und der De-Industrialisierung

Die Gemeinsamkeit dieser Theorieschulen liegt darin, dass aus ihnen jeweils eigenständige Hypothesen zur Erklärung von Staatstätigkeit im internationalen Vergleich oder im Zeitvergleich folgen. Neben Anwendungen in der Staatstätigkeitsforschung im engeren Sinne wurde die Schmidtsche Unterscheidung auch zur Analyse von Handlungen anderer Akteure als Regierungen, zur Beschreibung von Systemzuständen und zur vergleichenden Betrachtung von Outcomes wie Wachstum, Inflation und Arbeitslosigkeit genutzt (siehe beispielsweise die Beiträge in Obinger et al. 2003). In einem weiteren Sinne ließe sich also von Schulen der (vergleichenden) Politischen Ökonomie sprechen, deren Anwendungsbereich über die Analyse von Staatstätigkeit hinausreicht.

Theorien der sozioökonomischen Determination weisen dem sozioökonomischen Entwicklungsstand Erklärungskraft zu. Zu einflussreichen Theorien der sozioökonomischen Determination zählen Wagners Theorie der wachsenden Staatstätigkeit vor dem Hintergrund komplexer werdender kapitalistischer Gesellschaften (Wagner 1911/1970) sowie, auf höherem Abstraktionsgrad, die Marxsche Theorie der gesellschaftlichen Prägekraft des Stands der Produktivkräfte (Marx u. Engels 1848/1973, Abschn. I). MachtressourcenTheorien rücken Stand und Veränderung des Kräfteverhältnisses zwischen Schichten und Klassen ins Zentrum von Erklärungen. Ein eindrückliches Beispiel ist Korpis Erklärung der Genese moderner Wohlfahrtsstaaten und der schwedischen Sozialstaatsexpansion im Speziellen (Korpi 1978). In relativer Nähe hierzu bewegen sich Parteiendifferenztheorien, denen es aber nicht auf gesellschaftliche Kräfteverhältnisse allgemein, sondern vielmehr auf die parteipolitische Zusammensetzung von Regierungen ankommt (grundlegend Hibbs 1977, 1992). Politisch-institutionalistische Theorien betonen hingegen die Erklärungskraft unterschiedlicher institutionalisierter Spielregeln der politischen Systeme. Dazu zählen beispielsweise systematische Politikeffekte der Mehrheitsdemokratie in Abgrenzung zur Verhandlungsdemokratie (Lijphart 1999, Kap. 15, 16), der Zentral- 
bankunabhängigkeit (Busch 1995, S. 91-134) oder direktdemokratischer Institutionen (Schmidt 2008, Kap. 20; Wagschal u. Freitag 2007). Die fünfte hier unterschiedene Theorieschule fokussiert auf die Effekte der Internationalisierung der Güter-, Arbeits- und Finanzmärkte und deren ermöglichende oder behindernde Wirkung auf die Staatstätigkeit (Zohlnhöfer 2005); neben der Internationalisierung wurde die De-Industrialisierung reifer Volkswirtschaften als Determinante von Staatstätigkeit, insbesondere im Bereich der wohlfahrtsstaatlichen Politik, ausgemacht (Iversen u. Cusack 2000).

Diese Zusammenstellung ${ }^{1}$ existiert in unterschiedlichen Ausprägungen, und sie sollte nicht als abschließendes, sondern als ein je nach Anwendungsfall offenes Angebot verstanden werden. Auch existieren Theorien, deren Zuordnung zu den unterschiedenen Schulen nicht eindeutig ist. So wäre beispielsweise diskutabel, Parteiendifferenztheorien als Spezialfall von Machtressourcen-Theorien zu werten; ${ }^{2}$ es wäre denkbar, Theorien der Internationalisierung und der De-Industrialisierung unter die Theorien der sozioökonomischen Determination zu subsumieren; ${ }^{3}$ es wurden weit reichende Policy-Konsequenzen der Europäisierung nachgewiesen, hinter denen eine andere Logik steht als hinter Internationalisierungsprozessen, so dass bedenkenswert erscheint, Theorien supranationaler hoheitsstaatlicher Integration als eigenständige Theorieschule in die Unterscheidung aufzunehmen (grundlegend Scharpf 1999); und verschiedentlich wurde vorgeschlagen, Theorien des pfadabhängigen Wandels in der Liste von Schulen der Staatstätigkeitsforschung einen eigenständigen Platz einzuräumen. ${ }^{4}$

1 Bemerkenswert ist zudem, dass sich nahezu alle unterschiedenen Politikschulen anhand von Analysen wohlfahrtsstaatlicher Politik entwickelten, und dass sie - in der bei Schmidt angegeben Reihenfolge - eine zeitliche Abfolge beschreiben: Frühe Erklärungen der Genese des modernen Sozialstaats stellten auf funktionale Erfordernisse der Entwicklung der Industriegesellschaft ab (Wilensky u. Lebaux 1958, Kap. I-V), gefolgt von Machtressourcen- und Parteiendifferenztheorien (Hibbs 1977; Schmidt 1982, Kap. 2) und wiederum gefolgt von Studien zum prägenden Einfluss politisch-institutioneller Gefüge und Vetospieler-Konstellationen (Scharpf 1985; Schmidt 2002). Studien zum Einfluss der Internationalisierung und der De-Industrialisierung auf wohlfahrtsstaatliche Politik sind fast ausschließlich späteren Datums (Iversen u. Cusack 2000). Die „Spielarten des Kapitalismus“-Theorie, die ich nachfolgend als eigenständige Schule der vergleichenden Staatstätigkeitsforschung vorschlagen werde, hat sich insbesondere in der ersten Dekade der 2000er Jahre zu einer „Schule“ im engeren Sinne entwickelt.

2 Allerdings ist zu bedenken, dass gesellschaftliche Kräfteverhältnisse und die parteipolitische Zusammensetzung von Regierungen unabhängig voneinander variieren können. So können Rechtsparteien auch vor dem Hintergrund außergewöhnlich starker Gewerkschaftsbewegungen Regierungsbeteiligung erlangen, Gewerkschaften können auch vor dem Hintergrund stabiler Linksregierungen Einflussverlust erleiden.

3 Das gilt für die De-Industrialisierung mehr als für die Internationalisierung. Während die Schrumpfung des Industriesektors mit zunehmender sozioökonomischer Reife von Volkswirtschaften linear zuzunehmen scheint, variieren Grade an Internationalisierung unabhängig vom sozioökonomischen Entwicklungsstand.

4 Siehe beispielsweise Abschn. I.7 in Schmidt et al. (2007). Der besondere Reiz der Berücksichtigung der „Lehre von der Politikerblast“ liegt darin, dass im Fall der Anwendung von Regressionstechniken auch das Ausgangsniveau der abhängigen Variable bzw., im Fall gekreuzter Längs- und Querschnittsanalysen, die lagged dependent variable einer Theorieschule zugeordnet werden kann. Allerdings beschreibt die dahinter stehende Pfadabhängigkeitstheorie eher 
An der prinzipiellen Offenheit dieser Liste setzt die nachfolgende Argumentation an. Ich werde aufzeigen, dass insbesondere im englischsprachigen Raum seit den neunziger Jahren, vor allem aber in der laufenden Dekade eine neue Theorieschule herangewachsen ist, die als eigenständige Schule der vergleichenden Staatstätigkeitsforschung gewertet werden sollte: „Spielarten des Kapitalismus“ (nachfolgend mitunter: VoC, Varieties of Capitalism; alternative Bezeichnung: Produktionsregime-Ansatz). Die vorgeschlagene Einordnung dieser Schule in die vergleichende Staatstätigkeitsforschung mag zunächst verwundern. Niemand würde bestreiten, dass die „Spielarten des Kapitalismus“-Schule in den vergangenen Jahren eine der lebhaftesten Debatten der Politischen Ökonomie angestoßen und produktive Erkenntnisse insbesondere über die institutionellen Grundlagen wirtschaftlicher Wettbewerbsvorteile hervorgebracht hat (siehe stellvertretend für viele andere die Beiträge in Hall u. Soskice 2001b; Hancké et al. 2007a). Von Staatstätigkeit im engeren Sinne war in Hall u. Soskices "Introduction to Varieties of Capitalism" aber vergleichsweise wenig die Rede (Hall u. Soskice 2001a, Abschn. 1.7, 1.8). In den nachfolgenden Jahren indes wurden die Grundannahmen der ,Spielarten des Kapitalismus"-Schule, mitunter mit verblüffenden Ergebnissen, auf unterschiedliche Aspekte der Staatstätigkeit angewandt, und insbesondere zur vergleichenden Analyse des modernen Wohlfahrtsstaats entwickelte sich eine lebhafte, neuartige Diskussion.

Kurz: Sollte die Schmidtsche Unterscheidung von Schulen der vergleichenden Staatstätigkeitsforschung um eine weitere Schule ergänzt werden, wäre „Spielarten des Kapitalismus“ der nachdrücklichste Kandidat. Er erfüllt alle Kriterien einer solchen Schule: Aus ihm folgen Hypothesen, die sich auf keine der anderen Schulen, oder auf Kombinationen von ihnen, abbilden lassen; ${ }^{5}$ die Theorie ist, anders als beispielsweise funktionale Systemtheorien Luhmannscher Prägung, mit Bestandteilen der anderen Theorien zu politikfeldspezifischen Teiltheorien kombinierbar; und Originalität sowie die hohe Anzahl von Anwendungen auf Forschungsfragen der vergleichenden Policy-Forschung rechtfertigen die Wertung als eigenständige Theorieschule.

Um dies zu zeigen, werde ich nachfolgend in drei Schritten vorgehen. Zunächst spitze ich die Grundzüge des Theorieangebots problemorientiert und im Hinblick auf ihre Anwendung in der Politikanalyse zu. Im Anschluss daran zeige ich anhand von drei Beispielen, inwiefern aus „Spielarten des Kapitalismus“ eigenständige Hypothesen zu sowohl vergleichender Staatstätigkeitsforschung als auch, allgemeiner, zur vergleichenden Politischen Ökonomie folgen. Abschließend diskutiere ich die Implikationen für gegenwärtige und zukünftige Herausforderungen der vergleichenden Staatstätigkeitsforschung.

eine allgemeine Eigenschaft von Institutionen in Zeiten der friedlichen Entwicklung von Industrienationen, weniger hingegen eine Kausalität zur Erklärung von Staatstätigkeit, die sich einer "Schule“ im engeren Sinne zuordnen ließe. Ob sich eine Wertung als eigenständige Theorieschule empfiehlt, hängt letztlich vom Erkenntnisinteresse des Theorieanwenders ab.

5 Angaben zur Abgrenzung zur politisch-institutionalistischen Theorieschule, der VoC am ehesten zugeordnet werden könnte, erfolgen am Ende des Abschn. 2.1. 


\section{Sechs Grundannahmen und Perspektiven „Spielarten des Kapitalismus“-Schule}

„Spielarten des Kapitalismus“ erlangt seine distinkte Stellung als „Schule“ durch Setzung einer Anzahl von Grundannahmen und Einnahme von Perspektiven, die nachfolgend eingehender erläutert und im Hinblick auf ihren Erklärungswert für die Staatstätigkeitsforschung zugespitzt werden.

\subsection{Unternehmenszentrierung}

Das „Spielarten des Kapitalismus“-Konzept fokussiert auf Unternehmen als maßgebliche Akteure in Produktionsregimen und als entscheidende Empfänger wirtschaftspolitischer Regulierung. Diese Setzung unterscheidet VoC grundsätzlich von allen anderen Schulen der vergleichenden Staatstätigkeitsforschung (Hall u. Soskice 2001a, S. 6/7; Hall u. Thelen 2009, S. 8; kritisch: Amable u. Palombarini 2009, S. 126-129; Schmidt 2008, S. 311-313). Die theoriegeschichtlichen Ursachen dieser entscheidenden Grundintuition der VoC-Forschung werden deutlich, wenn man sich ihre Verwurzelung in der Korporatismusdiskussion der siebziger und achtziger Jahre vor Augen führt.

Spätestens seit den siebziger Jahren betrat die vergleichende Politikwissenschaft Terrain, das eigentlich die Wirtschaftswissenschaften für sich beanspruchten: nämlich, Erklärungen für die unterschiedlichen Grade an wirtschaftlichem Erfolg von Volkswirtschaften, gemessen anhand von Indikatoren wie dem Wirtschaftswachstum, der Inflation oder der Arbeitslosigkeit, bereitzuhalten. Das reine Marktmodell, so die starke These, war alternativen Formen der Steuerung der Wirtschaft nicht grundsätzlich überlegen. Ganz im Gegenteil erschien der Neokorporatismus, also die Durchorganisierung der Produktionsfaktoren Kapital und Arbeit in starken Verbänden und deren institutionalisierte Einbindung in die Politikproduktion, eine den Problemen reifer Volkswirtschaften adäquate Steuerungsform. Mit den Daten der siebziger Jahre ließ sich diese These plausibel bestätigen. Zahlreiche ländervergleichende Studien zeigten linear-inverse Zusammenhänge zwischen dem Grad an Neokorporatismus einerseits, und Inflation und Arbeitslosigkeit andererseits (Bruno u. Sachs 1985; Crepaz 1992; Crouch 1990; Czada 1983; Dell’ Aringa u. Lodovici 1992; Schmid 1993, 1982).

Dieses Ergebnis hielt aber nur, so lange sich die betrachteten Daten nicht zu weit in die achtziger oder gar neunziger Jahre hinein erstreckten. Bereits für die achtziger Jahre wich die Vermutung eines monotonen Zusammenhangs zwischen der Struktur der Verbändesysteme und deren quasi-staatlicher Einbindung in die Politikproduktion einerseits und der Leistungsfähigkeit der Ökonomien andererseits der These eines U-Kurven-förmigen Zusammenhangs, und zwar dahingehend, dass Marktsysteme und zentralisiert-korporatistische Systeme ebenbürtige, Mischsysteme hingegen schlechtere Ergebnisse hervorbrachten (Freeman 1988; Heitger 1987; Scarpetta 1996; in Bezug auf die Zentralisation der Lohnfindung: Calmfors u. Driffill 1988). Das Korporatismus-Konzept war nicht mehr geeignet, die Unterlegenheit des Marktmodells nachzuweisen. Dieses Problem aber ließ sich mit einer graduellen Umsortierung der Länder auf den gängigen KorporatismusSkalen und einer damit einhergehenden graduellen Anpassung des dahinter stehenden theoretischen Konzepts beheben. 
Nichts anderes tat Soskice (1990) in einer der ersten Darstellungen seiner Unterscheidung strategisch koordinierter und marktkoordinierter Ökonomien. Er zeigte auf, dass sich der von Calmfors und Driffill nachgewiesene U-Kurven-förmige Zusammenhang in einen monotonen Zusammenhang rücktransformierte, ${ }^{6}$ wenn man Japan und die Schweiz nicht als marktkoordinierte, sondern als strategisch koordinierte Ökonomien behandelte. Zudem wurde Deutschland, seinerzeit noch eine Ökonomie mit beispielgebendem Mix aus geringer Inflation und allenfalls auf mittlerem Niveau ausgeprägter Arbeitslosigkeit, von einem Mischtyp zum Paradefall einer koordinierten Ökonomie umklassifiziert. Wie musste das diese Umsortierung rechtfertigende Theoriekonzept aussehen? Erstens: Man musste sich von der Gewerkschaftsfixiertheit der Korporatismusdebatte lösen und stärker als bisher auf die Koordination auf Arbeitgeberseite schauen. Zweitens: Man musste auch dezentrale Koordinationsformen, die nicht über zentralisierte Verbändesysteme zustande kamen, als nichtmarktliche Koordination werten. Weder Japan noch die Schweiz verfügten über starke Gewerkschaften, und in Japan erfolgte arbeitgeberseitige Koordination über Industriegruppen statt über formale Verbände. Die logische Konsequenz lautete, dass sich der Fokus der Forschung von den in die Politik eingebundenen Verbänden ablösen und den Unternehmen zuwenden musste. So lässt sich die Genese des „Spielarten des Kapitalismus“-Konzepts als graduelle und von einer spezifischen makroökonomischen Datenlage getriebene Weiterentwicklung des Korporatismuskonzepts verstehen.

Das Neokorporatismus-Konzept wurde mitunter (Schmidt 1995, S. 584; Zohlnhöfer 2007, S. 161) der politisch-institutionalistischen Theorieschule zugerechnet, so dass sich die Frage stellt, ob nicht auch „Spielarten des Kapitalismus“ letztlich als politisch-institutionalistischer Ansatz gewertet werden sollte. ${ }^{7}$ Meines Erachtens würde diese Zuordnung mehr verdecken als erhellen. Die Nähe korporatistischer Erklärungen zu den politischinstitutionalistischen Ansätzen ergab sich aus der Staatsbezogenheit des Neokorporatismus, also der Tatsache, dass Politikproduktion als Aushandlungsdreieck zwischen Staat, Gewerkschaften und Arbeitgeberverbänden modelliert wurde. Im Neokorporatismus wurde das Verbändesystem damit faktisch zu einem Bestandteil des politischen Systems. Gerade dieser Theorieaspekt ist es aber, der bei dem Schritt vom Neokorporatismus zu „Spielarten des Kapitalismus“ verloren ging. Folgerichtig wird sich in Abschn. 3 zeigen, dass die Hypothesen, die VoC zur Erklärung von Staatstätigkeit generiert, mit gänzlich anderen, eigenen Kausalitäten operieren. Bei diesen Hypothesen geht es durchweg um Muster der Präferenzformierung auf der Mikro-Ebene, die mit korporatismus- oder beispielsweise vetospielertheoretischen Erklärungen nichts gemein haben.

6 Auch dieser Zusammenhang hielt nur für einen begrenzten Zeitraum. In jüngeren Darstellungen wird wiederum von einem U-förmigen Zusammenhang zwischen Grad an Koordiniertheit des Produktionsregimes einerseits und makroökonomischem Erfolg andererseits ausgegangen (Hall u. Gingerich 2004; vergleiche hierzu auch Abschn. 2.6).

7 Ich danke einem der anonymen Gutachter, der mich auf diesen möglichen Einwand aufmerksam machte. 


\subsection{Koordinationsmodi}

Was genau interessiert an den Unternehmen, die durch die oben skizzierten Weiterentwicklungen des Korporatismuskonzepts in das Zentrum der Aufmerksamkeit gerückt wurden? Es geht um die institutionalisierten Modi, mit denen sich Unternehmensleitungen untereinander und mit den Trägern der Ressourcen koordinieren, die für die Produktion gebraucht werden: mit Beschäftigten, Kunden und Zulieferern, Eigentümern, Kreditgebern und mit anderen Unternehmen. In dieser Hinsicht knüpft VoC an die Governance-Diskussion an, beschäftigt sich also mit Regelungsformen, die sich nicht unter den Begriff der hierarchischen Steuerung subsumieren lassen. Beispielsweise unterschieden sowohl Streeck u. Schmitter (1985) als auch Mayntz u. Scharpf (1995) vier typische Steuerungsarenen und ihnen zugehörige dominante Regelungsmodi: den Markt (Modus: unkoordinierte Konkurrenz), die Gemeinschaft (Modus: Solidarität), die Bürokratie (Modus: Hierarchie) und Verbände (Modus: Aushandlung); als fünfte Form trat in der Steuerungsdiskussion der achtziger und neunziger Jahre die Koordination in Netzwerken hinzu, die mal als Mischtyp zwischen den anderen Steuerungsformen, mal als eigenständige Regelungsform interpretiert wurde.

Bemerkenswert an der Aufnahme von Ergebnissen der Governance-Diskussion in das VoC-Konzept ist, dass sie mit einem radikalen Vereinfachungsschritt einhergeht: Es werden lediglich zwei Koordinationsmodi unterschieden, nämlich marktliche Koordination einerseits und alle Formen langfristiger, strategischer Koordination andererseits - gleichgültig, ob es sich um Koordination über Netzwerke handelt, ob Elemente der Hierarchie zwischen den Trägern der Koordination präsent sind, ob verbandliche Arenen zur Koordination genutzt werden oder ob sich Elemente von Reziprozität zwischen den beobachteten Einheiten nachweisen lassen. Kontroverse Diskussionen über die Heterogenität der als „koordinierte Marktökonomien“ zusammengefassten Ländergruppe (vergleiche Abschn. 2.5) wurzeln in dieser Verschiedenheit der Steuerungsformen, die unter dem Typus der strategischen Koordination zusammengefasst und dem Marktmechanismus gegenübergestellt werden.

\subsection{Institutionelle Sphären}

Die unterschiedlichen Koordinationsmodi werden in einer begrenzten Anzahl institutioneller Sphären (alternative Bezeichnung: Domänen) lokalisiert, wobei Anzahl und insbesondere Abgrenzung der Sphären in unterschiedlichen Darstellungen variieren. Insbesondere aber sind folgende vier Sphären zu nennen: Arbeitsbeziehungen, Unternehmensfinanzierung und -kontrolle (Corporate Governance), Ausbildungswesen sowie eine heterogene Kategorie, in der es um die Koordination zwischen Unternehmen geht und die sich auf Felder wie die Produktstandardisierung, den Technologietransfer und die allgemeine Wettbewerbspolitik bezieht. In einigen Darstellungen wird die interne Struktur der Unternehmen (z. B.: institutionelle Separierung der Leitungsorgane in Vorstände und Aufsichtsräte, Unternehmensmitbestimmung) als eigenständige, von Unternehmenskontrolle und Arbeitsbeziehungen abzugrenzende Domäne behandelt (Hall u. Soskice 2001a, S. 24).

Aus institutionentheoretischer Perspektive ist von besonderem Interesse, ob das Verhältnis dieser Sphären zueinander als heterarchisch modelliert wird, oder eine Leitdomäne 
existiert, deren Struktureigenschaften in asymmetrischer Weise prägend auf benachbarte Domänen einwirken (vergleiche Abschn. 2.4 zur funktionalen Komplementarität). Mit dem Gedanken einer im Zeitverlauf wechselnden Hierarchie zwischen den Sphären von Produktionsregimen operiert beispielsweise die französische Regulationsschule. Dieser Sicht zufolge fungierten die Institutionen der Arbeitsbeziehungen im Fordismus als Leitsphäre, wurden im Lauf der zweiten Hälfte des zwanzigsten Jahrhunderts aber von einer Dominanz der Wettbewerbspolitik über die Struktureigenschaften anderer Domänen abgelöst (Aglietta 1976, S. 383; Amable u. Petit 2001, S. 10; Boyer 1990, S. 108; Boyer u. Saillard 2002, S. 39). Bei Boyer (1998, 2000) findet sich die These, dass sich die Institutionen des Finanzmarktregimes im späten zwanzigsten Jahrhundert in eine zunehmend dominante Position schoben und Anpassungen der institutionellen Verfasstheit anderer Sphären erzwangen. Eine explizite Thematisierung solch institutioneller Hierarchien findet sich im „Spielarten des Kapitalismus“-Konzept nicht.

Implizit hingegen finden sich auch in der VoC-Schule Zuschreibungen, die ein hierarchisches Verhältnis zwischen den institutionellen Sphären nahe legen. "Financial deregulation", schreiben Hall u. Soskice (2001a, S. 64), "could be the string that unravels coordinated market economies". Dies würde für eine übergeordnete Stellung des Finanzmarktregimes sprechen. Allerdings findet gerade die Beziehung zwischen Unternehmen und Investoren in jüngeren Diskussionsbeiträgen aus dem VoC-Umfeld kaum noch explizite Beachtung (Hall u. Thelen 2009; Iversen 2005), so dass sich anstelle der Frage einer etwaigen institutionellen Dominanz dieser Sphäre sogar eher die Frage stellt, ob sie im Konzept überhaupt noch zur Erklärung von Verhaltensweisen der Unternehmen und der Politik sowie Outcomes gebraucht wird. Indes hat sich eine andere Sphäre in das Zentrum der Aufmerksamkeit geschoben: die Domäne der Humankapitalproduktion. Diesen Sachverhalt werde ich in Abschn. 3 anhand der drei Beispiele zur policybezogenen Erklärungskraft der „Spielarten des Kapitalismus“-Schule illustrieren. ${ }^{8}$

\subsection{Institutionelle Komplementarität}

Neben der Unternehmenszentrierung ist die herausragende Bedeutung der institutionellen Komplementarität ein distinktes Merkmal der „Spielarten des Kapitalismus“-Schule. Sie interessiert sich weniger für unabhängig voneinander bestehende, kumulierte Wirkungen von Institutionen aus unterschiedlichen Sphären, als vielmehr für systematische Interaktionseffekte zwischen ihnen. Institutionelle Komplementarität liegt vor, wenn die Funktionalität einer Institution von der Präsenz anderer Institutionen abhängt. ${ }^{9}$ Diese an sich einfache Setzung der Theorie hat weit reichende Konsequenzen für das Denken über

8 In Abschn. 3 wird auch deutlich, dass eine weitere Sphäre inzwischen als eine hinsichtlich ihrer Funktionalität für Unternehmen den anderen Domänen ebenbürtige Sphäre behandelt wird: der Wohlfahrtsstaat.

9 Dabei handelt es sich um eine enge Definition institutioneller Komplementarität. Mitunter und m.E. nicht zielführend wird der Begriff auch zur Bezeichnung der Ähnlichkeit von Struktureigenschaften von Institutionen gebraucht (treffender: Kohärenz, siehe hierzu Abschn. 2.6). Vergleiche hierzu ausführlich die in Ausgabe 2/2005 der "Socio-Economic Review" geführte Debatte über institutionelle Komplementarität. 
Institutionen. Das sei anhand eines Beispiels erläutert, das bereits auf die in Abschn. 3 erfolgende Diskussion der Staatstätigkeit in unterschiedlichen Produktionsregimen hinausweist. Darin geht es um die Komplementarität zwischen den Institutionen der Humankapitalproduktion und dem Kündigungsschutz. ${ }^{10}$

Aus Sicht der Neoklassik bürdet Kündigungsschutz Unternehmen Kosten auf, die sich zu den primären Personalkosten hinzuaddieren, die faktischen Personalkosten somit über das markträumende Gleichgewichtsniveau heben und deshalb in Unterbeschäftigung münden müssen. Vertreter der VoC-Schule halten dem entgegen, diese Betrachtung vernachlässige Interaktionseffekte zwischen Institutionen. Angenommen sei ein Unternehmen, dessen Wettbewerbsvorteile bei der Produktion spezialisierter Qualitätsprodukte auf der Verfügbarkeit unternehmensspezifischen Humankapitals beruhen. Was konkret bedeutet: Die zur Produktion notwenigen Fertigkeiten lassen sich auf dem externen Arbeitsmarkt nicht einkaufen, sondern müssen im Unternehmen erbracht werden. Die betroffenen Humankapitalträger haben im Fall von Kündigungen nur geringe Chancen, ein vergleichbares Einkommen in anderen Unternehmen oder sogar Sektoren zu erzielen; ihre Humankapitalinvestitionen sind sunk cost und entwerten sich bei dem Versuch, sie auf andere Unternehmen zu übertragen. Dieses Unternehmen ist somit auf die Bereitschaft älterer Arbeitnehmer angewiesen, ihre Fertigkeiten im Rahmen betrieblicher Ausbildungsgänge an jüngere Beschäftigte weiterzugeben. Warum sollten sie dies tun, wenn sie befürchten müssten, im Anschluss durch den jüngeren, potenziell produktiveren Beschäftigten ersetzt zu werden? Die Bereitschaft, eigene unternehmensspezifische Qualifikationen effektiv an andere zu übertragen, so die Schlussfolgerung, setzt glaubhaften Schutz vor Kündigungen voraus. Was aber, wenn man dieses Gedankenmodell von der Unternehmensebene auf die Ebene einer Volkswirtschaft überträgt, also eine „Spielart des Kapitalismus“ annimmt, deren Wettbewerbsvorteile in der internationalen Arbeitsteilung auf friktionsloser Generierung spezifischen Humankapitals beruht? Dann gewinnt der Kündigungsschutz als Institution eine produktionsbezogene, effizienztechnische Funktionalität.

Bemerkenswert ist nun, dass die oben skizzierte Argumentation nicht mit Annahmen operiert, die der wirtschaftswissenschaftlichen Neoklassik widersprechen würden. Es ist lediglich die Einsicht in - wenn man so will - „Querwirkungen“ von Institutionen, die den Kündigungsschutz plötzlich nicht mehr als sozialpolitisch vielleicht wünschenswertes, wirtschaftlich aber gewiss nicht effizientes Instrument erscheinen lässt. Vielmehr, so das Argument, hängt es von den anderen institutionellen Elementen des Produktionsregimes $\mathrm{ab}$, ob eine Institution wie der Kündigungsschutz einen funktionalen Beitrag zu Produktion und Wettbewerbsfähigkeit leistet oder nicht. Ähnliches lässt sich für weitere Institutionen durchdeklinieren, die ihre Funktionalität auf den ersten Blick lediglich im Sinne des Arbeitnehmerschutzes entfalten, nicht aber im Sinne eines produktiven Beitrags zur Wettbewerbskraft von Volkswirtschaften: so beispielsweise für Elemente der Sozialpolitik (vergleiche Abschn. 3.1), der zentralisierten Lohnfindung oder der Mitbestimmung. Allgemein formuliert: Die Einsicht in die Eigenschaft institutioneller Komplementarität legt nahe, dass die Funktionalität der Institutionen von Produktionsregimen niemals isoliert bestimmt werden kann, sondern sich erst aus dem Zusammenspiel mit anderen Institutionen ergibt.

10 Das meinem Eindruck nach besonders anschauliche Beispiel stammt aus einem nicht publizierten Vortrag von Wolfgang Streeck. 


\subsection{Nationale Spielarten des Kapitalismus}

Mit Hilfe der bis hier dargestellten Konzepte lassen sich nationale Spielarten des Kapitalismus unterscheiden und zu Gruppen oder Rangfolgen sortieren. Hall und Soskice operieren mit der binären Unterscheidung zwischen liberalen Marktökonomien (liberal market economies, LMEs) und koordinierten Marktökonomien (coordinated market economies, CMEs) und klassifizieren zudem einige Länder als Mischtypen (siehe Tab. 1, Spalte 2). Wegen der in Abschn. 2.2 angesprochenen Heterogenität der in CMEs dominanten Steuerungsmodi haben einige Autoren die Ausweitung der Typologie auf mehr als zwei Ausprägungen vorgeschlagen. So unterscheidet Schmidt (2002) drei Kapitalismustypen: Marktkapitalismus, koordinierten Kapitalismus (in ihrer Terminologie: managed capitalism) und den Staatskapitalismus französischer Prägung. Hancké et al. (2007b, S. 24-28) greifen diesen Vorschlag auf, spalten aus der Gruppe der CMEs aber eine weitere Unterkategorie ab, die sie durch die Kombination von in starken Verbänden organisierten Firmen und einem industriepolitisch aktiven Staat charakterisiert sehen und der sie Italien, Spanien und einige osteuropäische Länder zuordnen. ${ }^{11}$ Amable (2003, Kap. 5) unterscheidet fünf Typen: den marktbasierten, den asiatischen, den sozialdemokratisch-nordischen, den mediterranen sowie den kontinentaleuropäischen Kapitalismus (Spalte 3).

Eine andere Möglichkeit besteht darin, nach dem Vorbild der Korporatismus-Skalen der siebziger bis neunziger Jahre zwei Idealtypen zu definieren und die vorgefundenen Realtypen auf einer Achse zwischen ihnen zu verorten. Wegen ihrer Eindimensionalität bleiben diese Skalen beispielsweise gegenüber Amables Typologisierung notwendig unterkomplex. Ihre Vorteile liegen indes darin, dass sie den Anschein der Möglichkeit eindeutiger kategorialer Zuordnung vermeiden und dass sie als metrische Skalen in der quantitativen Politikforschung einsetzbar sind. Tabelle 1 zeigt drei solche Messversuche: Hall und Gingerichs Index des Ausmaßes an strategischer Koordination in Ökonomien (Spalte 4), Hicks und Kenworthys Kooperationsindex (Spalte 5) sowie Höpners Skala des organisierten Kapitalismus (Spalte 6). Der Index von Hall u. Gingerich (2004) fasst Daten zu den Rechten von Minderheitsaktionären, zum Anteil des Streubesitzes an den Eigentümerstrukturen großer Unternehmen, zur Kapitalisierung der Aktienmärkte, zur Koordination im Bereich der Lohnaushandlung und zur Arbeitsmarktfluktuation zusammen. Hicks u. Kenworthys (dies. 1998) Index wurde aus Rohdaten zur Langfristigkeit der Zulieferer- und Abnehmerbeziehungen, zur Kooperation zwischen Unternehmen in Bereichen wie Ausbildung und Technologietransfer, zum Ausmaß an Gruppenarbeit sowie zur ressortübergreifenden Projektarbeit in Unternehmen gebildet. Höpners (2007) Index des organisierten Kapitalismus besteht aus vier Einzelkomponenten: Aktienanteile großer Unternehmen in Eigentümerschaft von öffentlichen Gebietskörperschaften und von anderen Großunternehmen (Unternehmensverflechtungen), Arbeitnehmermitbestimmung auf der Ebene der Leitungsorgane, Organisationsquoten der Arbeitgeberverbände sowie entsprechende Quoten der Gewerkschaften.

11 Leider präsentieren Hancké et al. (2007b, S. 24-28) ihren Vorschlag nur konzeptionell, ohne ihn systematisch auf die OECD-Länder anzuwenden. 
Tab. 1: Länderskalen in der „Spielarten des Kapitalismus“-Forschung: 20 OECD-Länder, unterschiedliche Bezugszeiträume

\begin{tabular}{|c|c|c|c|c|c|}
\hline & $\begin{array}{l}\text { Hall und Soski- } \\
\text { ces kategoriale } \\
\text { Zuordnung, } \\
\text { 1990er Jahre }\end{array}$ & $\begin{array}{l}\text { Amables } \\
\text { kategoriale } \\
\text { Zuordnung, } \\
\text { 1990er Jahre } \\
\end{array}$ & $\begin{array}{l}\text { Hall und Ginge- } \\
\text { richs Koordinati- } \\
\text { ons-Index, 1990er } \\
\text { Jahre }\end{array}$ & $\begin{array}{l}\text { Hicks und Kenwort- } \\
\text { hys Kooperations- } \\
\text { Index, 1960er bis } \\
\text { 1980er Jahre }\end{array}$ & $\begin{array}{l}\text { Höpners Index des } \\
\text { organisierten Kapi- } \\
\text { talismus, 1990er und } \\
\text { 2000er Jahre }\end{array}$ \\
\hline Australien & 1 & 1 & 0,36 & 0,00 & $-0,14$ \\
\hline Belgien & 3 & 3 & 0,74 & 0,25 & 0,34 \\
\hline Dänemark & 3 & 4 & 0,70 & 0,25 & 0,71 \\
\hline Deutschland & 3 & 3 & 0,95 & 0,39 & 0,94 \\
\hline Finnland & 3 & 4 & 0,72 & 0,38 & 1,28 \\
\hline Frankreich & 2 & 3 & 0,69 & 0,03 & $-0,36$ \\
\hline Großbritannien & 1 & 1 & 0,07 & 0,02 & $-0,93$ \\
\hline Irland & 1 & 3 & 0,29 & 0,00 & $-0,48$ \\
\hline Italien & 2 & 5 & 0,87 & 0,42 & 0,05 \\
\hline Japan & 3 & 2 & 0,74 & 0,92 & $-1,17$ \\
\hline Kanada & 1 & 1 & 0,13 & 0,00 & $-1,36$ \\
\hline Neuseeland & 1 & - & 0,21 & 0,00 & 0,23 \\
\hline Niederlande & 3 & 3 & 0,66 & 0,02 & 0,80 \\
\hline Norwegen & 3 & 3 & 0,76 & 0,38 & 0,70 \\
\hline Österreich & 3 & 3 & 1,00 & 0,25 & 1,87 \\
\hline Portugal & 2 & 5 & 0,72 & - & $-0,54$ \\
\hline Schweden & 3 & 4 & 0,69 & 0,32 & 1,20 \\
\hline Schweiz & 3 & 3 & 0,51 & 0,25 & $-1,10$ \\
\hline Spanien & 2 & 5 & 0,57 & - & $-0,09$ \\
\hline USA & 1 & 1 & 0,00 & 0,05 & $-1,93$ \\
\hline
\end{tabular}

Spalte 1: Ländername

Spalte 2: Kategoriale Länderzordnung nach Hall u. Soskice. 1=Liberale Marktökonomie (LME); 2=Mischtyp; 3 =Koordinierte Marktökonomie (CME). Bezugszeitraum: 1990er Jahre. Quelle: Hall u. Soskice (2001a, S. 19-21)

Spalte 3: Kategoriale Zuordnung nach Amable. 1=Marktbasierter Kapitalismus; 2=asiatischer Kapitalismus; 3 =kontinentaleuropäischer Kapitalismus; 4 =sozialdemokratischer Kapitalismus; 5 =mediterraner Kapitalismus. Bezugszeitraum: 1990er Jahre. Quelle: Amable (2003, S. 173)

Spalte 4: Index der strategischen Koordination in Produktionsregimen nach Hall u. Gingerich. Hohe Werte indizieren ein hohes Ausmaß an strategischer Koordination. Kombinierter Index aus sechs Einzelskalen: (1) Rechte von Minderheitsaktionären; (2) Anteil von Aktien in Streubesitz; (3) Marktkapitalisierung börsennotierter Unternehmen; (4) Ausmaß an Lohnkoordination; (5) Grad an Zentralisierung der Lohnkoordination; (6) Arbeitsmarktfluktuation. Bezugszeitraum: 1990er Jahre. Quelle: Hall u. Gingerich (2004, S. 14)

Spalte 5: Index der Kooperation innerhalb und zwischen Unternehmen nach Hicks und Kenworthy. Hohe Werte indizieren ein hohes Ausmaß an Kooperation. Kombinierter Index aus vier Einzelskalen: (1) Langfristigkeit der Beziehungen zwischen Produzenten, Zulieferern und Abnehmern; (2) Kooperation zwischen Unternehmen in den Bereichen Ausbildung, Forschung und Entwicklung, Standardsetzung, Technologietransfer; (3) Gruppenarbeit in den Unternehmen; (4) Bereichsübergreifende Projektarbeit in den Unternehmen. Bezugszeitraum: 1960er bis 1990er Jahre. Quelle: Hicks u. Kenworthy (1998, S. 1649)

Spalte 6: Index des organisierten Kapitalismus nach Höpner. Hohe Werte indizieren ein hohes Ausmaß an Organisiertheit. Kombinierter Index aus vier Einzelskalen: (1) Aktienanteile in Besitz öffentlicher Gebietskörperschaften und Unternehmen; (2) Arbeitnehmermitbestimmung auf Ebene der Leitungsorgane von Unternehmen; (3) Organisationsquote Arbeitgeberverbände; (4) Organisationsquote Gewerkschaften. Bezugszeitraum: 1990er und 2000er Jahre. Quelle: Höpner (2007, S. 14) 


\subsection{Effizienz und institutioneller Wandel}

Aus einem Vergleich der für die deutsche und die amerikanische Ökonomie typischen Innovationsmuster leiten Hall u. Soskice (2001a, S. 36-44) die These ab, dass es kohärent liberalen und kohärent koordinierten Produktionsregimen in besonderem Maße gelingt, komparative Vorteile in der internationalen Arbeitsteilung zu erwirtschaften, indem sie systematisch bestimmte Innovationstypen unterstützen: Inkrementelle Innovationen (zum Beispiel: im Maschinenbau) im Fall der koordinierten Ökonomien, radikale Innovationen (zum Beispiel: in der Informations- und Biotechnologie) im Fall der liberalen Ökonomien (kritisch: Taylor 2004). Hall u. Gingerich (2004) untersuchen Zusammenhänge zwischen dem Grad an Koordiniertheit von Ökonomien und Wachstumsraten der siebziger, achtziger und neunziger Jahre. Unter statistischer Kontrolle für zahlreiche weitere Variablen zeigen sie einen U-Kurven-förmigen Zusammenhang dahingehend, dass kohärent koordinierte und kohärent liberale Ökonomien höhere Wachstumsraten erwirtschaften als Mischtypen. Kohärenz, so die These, erlaubt die wirtschaftliche Ausschöpfung der Potenziale institutioneller Komplementarität. Demnach erscheint vorteilhaft, sich an den äußersten Rändern der Hall-Gingerich-Skala (Tab. 1, Spalte 4) zu befinden.

Im Hinblick auf politische Implikationen der VoC-Perspektive ist entscheidend, dass aus den Überlegungen zur Überlegenheit kohärenter Produktionsregime eine These über langfristigen institutionellen Wandel fließt. Denn halten die Überlegungen zu den Wirkungen der Kohärenz, dann wäre zu erwarten, dass wirtschaftliche und politische Eliten auf ökonomische Schocks und auf Wettbewerbsverschärfungen mit Reformen zur Steigerung der Kohärenz reagieren - und damit im Ergebnis höchst verschieden: mit weiterer Liberalisierung im Fall der ohnehin bereits auf wirtschaftsliberalen Grundsätzen beruhenden LMEs, mit der Beseitigung von Störungen strategischer Koordination im Fall der CMEs. Im Ergebnis, so diese wegen ihres kruden Funktionalismus wohl umstrittenste These der VoC-Schule (eindrücklich: Streeck 2009), wäre anzunehmen, dass sich Produktionsregime im Lauf ihrer Evolution immer stärker den kohärenten Enden der Koordinations-Skala annähern: "[N]ations with a particular type of coordination in one sphere of the economy should tend to develop complementary practices in other spheres as well", schreiben Hall u. Soskice (2001a, S. 17).

Diese weitreichende These kontrastiert in bemerkenswerter Weise mit den Prognosen führender Politökonomen des zwanzigsten Jahrhunderts über die langfristigen Entwicklungsdynamiken moderner Industriegesellschaften (ausführlich: Höpner et al. 2009, Abschn. 2). So unterschiedliche Autoren wie Keynes (1926), Schumpeter (1942/1950) und Olson (1982) gingen davon aus, den Wirtschaftssystemen liberaler Demokratien sei ein evolutionärer Trend der Entfernung von Marktprinzipien eigen. Das Gegenteil erwarteten evolutionäre Effizienztheorien: Der Markt, so diese Sicht, werde sich als effizientester Allokationsmechanismus langfristig durchsetzen (North u. Thomas 1973). ,Spielarten des Kapitalismus“" prognostiziert hingegen keine konvergente, langfristige Entwicklungsdynamik, sondern eine über die Zeit erfolgende Verfestigung unterschiedlicher Entwicklungspfade, in der Terminologie von Soskice (1999, S. 123): "bifurcated convergence".

Anzumerken bleibt, dass der Funktionalismus der bifurcated convergence-These auch von Autoren zurückgewiesen wurde, die der VoC-Schule im engeren Sinne zugerechnet werden können (siehe beispielsweise Hancké et al. 2007b, S. 12-14; vergleiche auch 
Höpner 2005; Howell 2003; Streeck 2005 und viele andere). Die These hat zwei hohe Plausibilitätshürden zu nehmen. Zum einen, dass die behaupteten Effizienzwirkungen der Kohärenz tatsächlich in spürbarer Stärke vorliegen. Und zum anderen, dass sich diese Wirkungen tatsächlich in Schubkräfte institutionellen Wandels übersetzen und stärker wirken als alle anderen vergleichbaren Schubkräfte, deren Mechanismen im Zentrum anderer Schulen der vergleichenden Staatstätigkeitsforschung stehen; dass Dynamiken institutionellen Wandels also tatsächlich zuvörderst wirtschaftlichen Effizienzgesichtspunkten folgen. "[T]he presence of one set of institutions cannot dictate the presence of a specific set of other institutions, even if the two are complementary", stellt Hall (2005, S. 375) vier Jahre nach Erscheinen der "Introduction to Varieties of Capitalism" als Ergebnis einer Debatte zur institutionellen Komplementarität klar. In seinem Beitrag zum Band "Beyond Varieties of Capitalism" geht Hall (2007) von Liberalisierungsprozessen sowohl in CMEs als auch in LMEs aus und sieht den Mehrwert der VoC-Perspektive vor allem in der Spezifizierung unterschiedlicher, von den Ausprägungen der Produktionsregime determinierter Liberalisierungspfade.

Bereits auf diesem hohen Abstraktionsniveau also werden Hypothesen zu typischen Fragestellungen der vergleichenden Staatstätigkeitsforschung generiert. Wie ich nachfolgend zeigen werde, wurde das „Spielarten des Kapitalismus“-Konzept zudem zur Generierung politikfeldspezifischer Hypothesen zur Genese von Staatstätigkeit genutzt, die über eine strikte Mikrofundierung verfügen und über die funktionalistische Zuschreibung von Politikmustern zu vermeintlichen Effizienzwirkungen hinausgehen.

\section{3 ,Spielarten des Kapitalismus“ als Quelle von Hypothesen zur vergleichenden Staatstätigkeitsforschung}

Inwiefern also fließen aus dem VoC-Konzept spezifische Hypothesen zur Erklärung von Staatstätigkeit? Die drei nachfolgend skizzierten Beispiele haben gemein, dass sie nach den policy-Wirkungen des Sets komplementärer Institutionen fragen, das auf die Ausbildung unternehmens- oder sektorspezifischen Humankapitals hinwirkt. Den Grundannahmen des Konzepts folgend, werden diese Institutionen nicht nur in der Sphäre der Humankapitalproduktion im engeren Sinne verortet, sondern auch in den ,benachbarten“ Sphären der Produktionsregime, also namentlich des Wettbewerbsregimes, der Arbeitsbeziehungen und der Unternehmenskontrolle. Gleichwohl bleibt festzuhalten, dass alle drei Beispiele die Voraussetzungen der Humankapitalbildung in das Zentrum der Überlegungen rücken. Implizit erlangt diese Domäne, trotz der eigentlich heterarchischen Modellierung des Verhältnisses der Sphären zueinander, damit den Charakter einer Leitdomäne (vergleiche die Diskussion in Abschn. 2.3).

\subsection{Die revisionistische Wohlfahrtsstaatsdebatte}

Bereits in Abschn. 2.4 wurde am Beispiel des Kündigungsschutzes eine Hypothese zu den produktionsbezogenen Wirkungen CME-typischer Institutionen entwickelt. Der Kündigungsschutz, so lautete die Hypothese, leistet einen funktionalen Beitrag zur Bereitschaft von Unternehmen und Beschäftigten, in unternehmensspezifisches Humankapital zu 
investieren. Nehmen wir nun in leichter Modifikation des Modells an, das vom Unternehmen benötigte Humankapital sei zwar nicht gänzlich unternehmensspezifisch, aber sektorspezifisch. Es sei im Prinzip transferierbar, der hierfür in Frage kommende Pool an Unternehmen sei aber klein und folglich müsse im Fall von Kündigungen mit einer im Vergleich zu generellen Skills längeren Phase der Sucharbeitslosigkeit gerechnet werden. Warum sollten die Beschäftigten in die Ausbildung eines solchen, mit hohem Entwertungsrisiko behafteten Humankapitals investieren, wo ihnen doch prinzipiell auch Wege zur Ausbildung genereller Fertigkeiten offen stehen? Müsste eine Volkswirtschaft, die aufgrund ihrer Stellung in der internationalen Arbeitsteilung auf die Ausbildung sektorspezifischer Fertigkeiten angewiesen ist, nicht latent mit Problemen der Unterversorgung mit dem benötigten Humankapital konfrontiert sein, und müsste der Staat den betroffenen Beschäftigten, um die Bereitschaft in die entsprechenden Investitionen zu fördern, im Fall der Arbeitslosigkeit nicht statusbezogene, also vom letzten erzielten Arbeitsentgelt abhängige Lohnersatzleistungen garantieren? Und wenn das so wäre: Sollten dann nicht auch die Arbeitgeber des hochproduktiven Exportsektors ein Interesse an der Gewährung solcher Sozialleistungen haben?

Für diese Überlegungen gilt, was bereits in Abschn. 2.4 für die Hypothesen zum Kündigungsschutz herausgestellt wurde: Sie beruhen nicht auf Prämissen, die neoklassischen Annahmen grundsätzlich widersprechen würden. Es ist allein die systematische Berücksichtigung der institutionellen Komplementarität (der „Querwirkung“ von Institutionen), die die Perspektive auf Institutionen wie die statusbezogene Sozialpolitik grundsätzlich verändert. Mit weit reichenden Konsequenzen nicht nur für die neoklassische Beurteilung von Kündigungsschutz und Sozialpolitik, sondern auch für die politikwissenschaftliche Machtressourcen-Theorie, die den Wohlfahrtsstaat vor allem als Resultat der relativen Machtstellung der Arbeiterklasse interpretierte und seine „dekommodifizierende“ Wirkung betonte, was, im Sinne eines positiven Rückkopplungseffekts, den Wohlfahrtsstaat seinerseits als Machtressource der Arbeiterklasse erscheinen ließ (Esping-Andersen 1990; Korpi 1978). Die implizite Annahme lautete hierbei, die Arbeitgeber seien Gegner des Wohlfahrtsstaats, hätten seine Genese zu verhindern gesucht und würden ihn (wieder) abschaffen, würden ihre Machtressourcen das zulassen. Diese Annahme wird durch die „revisionistische Wohlfahrtsstaatsdebatte“ hinterfragt. Ist der moderne Wohlfahrtsstaat, so ließe sich provokant fragen, am Ende ein Instrument von und für Arbeitgeber?

Das skizzierte Argument wird auf zwei Arten geführt und mit Empirie unterfüttert. In der historisch-genetischen Variante untersuchen Autoren wie Mares (2000, 2001, 2003) und Swenson (2002, 2004) entscheidende Wendepunkte bei der Entstehung moderner Wohlfahrtsstaaten und zeigen auf, dass die Haltungen der Arbeitgeber nicht von monolithischer Gegnerschaft geprägt waren. Beide Autoren argumentieren mit großer Vorsicht und warnen vor der Fehlinterpretation, der Wohlfahrtsstaat sei funktional und genetisch als Arbeitgeberinstrument zur Generierung von Wettbewerbsvorteilen zu verstehen. Swenson geht es um sektoral begrenzte, klassenübergreifende Koalitionen als Grundlage wohlfahrtsstaatlicher Politik in den USA und Schweden (Swenson 2002) sowie, in einem parallelen Argument, um die machtpolitischen Determinanten der Zentralisation der schwedischen und dänischen Tarifsysteme (Swenson 1991). Mares argumentiert noch zurückhaltender und stellt am Beispiel Deutschlands heraus, dass dominante Fraktionen des Kapitals - ihren ursprünglichen, ,vorstrategischen“ Präferenzen folgend 
- in den zwanziger Jahren tatsächlich zunächst gegen die Einführung der allgemeinen Arbeitslosenversicherung votierten. Um aber die aus ihrer Sicht schlechtesten Ergebnisse zu verhindern, ließen sich Unternehmen aus den hochproduktiven Exportsektoren auf Kompromisse ein und wurden so zu potenziellen Verbündeten der Reformflügel der Arbeiterbewegung. Die agenda setter und Architekten der Reformen, so stellt Mares heraus, waren keine Arbeitgeber, sondern Reformpolitiker, denen es gelang, auf Grundlage „nachstrategischer“, zweiter Präferenzen fragile Kompromisse zu schmieden und die sozioökonomischen Akteure auf diese zu verpflichten (Mares 2003).

Im Zentrum der revisionistischen Wohlfahrtsstaatsdebatte steht aber vor allem die mikrofundierte, wählerzentrierte Variante, die von Iversen und einigen Mitautoren vertreten wird (Estévez-Abe et al. 2001; Iversen 2005; Iversen u. Soskice 2001; Iversen u. Stephens 2008). Im Kern geht es dabei um eine Modifikation des Meltzer/RichardModells, das vom Einkommen des Wählers auf die Präferenz gegenüber staatlicher Sozialpolitik schließt und besagt, dass Wähler bis zum Medianeinkommen Befürworter von Umverteilung sind, Wähler oberhalb des Medianeinkommens hingegen Gegner (Meltzer u. Richard 1981). ${ }^{12}$ Diese Interpretation, so der Einwand, modelliere Einstellungen gegenüber Umverteilung im Prinzip korrekt, ignoriere aber die Versicherungsfunktion des Wohlfahrtsstaats, genauer: seine Schutzfunktion in Bezug auf nur begrenzt über Unternehmensgrenzen hinweg transferierbares Humankapital. Beide Funktionen, Umverteilung und Absicherung, seien im Wohlfahrtsstaat untrennbar verknüpft, und ebenso wie die Verfügung über physisches Kapital die Präferenzen gegenüber Umverteilung determiniere, präge die Verfügung über Humankapital die Präferenzen gegenüber der Versicherungsfunktion des Wohlfahrtsstaats. Wähler mit unternehmens- oder sektorspezifischem Humankapital, so zeigen Iversen u. Soskice (2001, S. 883-886) anhand von Umfragedaten, weisen auch dann noch eine Präferenz für Umverteilung auf, wenn man es aufgrund ihrer Einkommenshöhe nicht mehr erwarten würde. Die Autoren schließen: In Produktionsregimen, deren komplementäre Institutionen auf die Ausbildung spezifischen Humankapitals hinwirken, konkurrieren die großen Parteien um einen Medianwähler, der „pro Sozialstaat“ ist - und entwickeln deshalb eine dezidierte wohlfahrtsstaatliche Programmatik. Im Ergebnis wird die institutionelle Ausgestaltung des Produktionsregimes über den Umweg des Grads an Humankapitalspezifität zum Prädiktor für wohlfahrtsstaatliche Politik. ${ }^{13}$

12 Daraus folgt die Erwartung, dass Umverteilung in einer perfekten Mehrheitsdemokratie so lange fortgesetzt wird, wie der Medianwähler durch Erhöhung seines Einkommens davon profitiert.

13 Im Prinzip setzt diese Argumentation keine Annahmen zu den Arbeitgeberpräferenzen gegenüber Sozialpolitik voraus. Allerdings stehen die Argumente von Mares und Swenson einerseits und Iversen und Mitautoren andererseits auch nicht im Widerspruch zueinander. Sie verdichten sich zu einer These über den Beitrag statusbezogener Sozialpolitik zur Funktionsweise von Produktionsregimen, die auf der Ausbildung von spezifischem Humankapital beruhen, und damit auf einer Zurückweisung, mindestens aber Relativierung klassentheoretischer Interpretationen. Vergleiche hierzu kritisch Korpi (2006) sowie das Symposium zu Iversens "Capitalism, Democracy, and Welfare" (2005) in Ausgabe 3/2006 der Zeitschrift "Labor History", hier insbesondere Iversen (2006), Kitschelt (2006); Stephens (2006). Einen (frühen) Vergleich arbeitgeberzentrierter und machtressourcentheoretischer Perspektiven bietet Pierson (2000). 


\subsection{Geschlechtersegregation}

Das zweite Beispiel bezieht sich genau genommen nicht auf policies, sondern auf outcomes, die ihrerseits Problemkonstellationen herbeiführen, an denen sich Staatstätigkeit abarbeiten muss. Das Argument verknüpft auf verblüffende Weise Literaturstände, die auf den ersten Blick weit voneinander entfernt erscheinen: die Literaturen über Produktionsregime und Geschlechtersegregation. Die Vertreter des Arguments legen nahe, mit den Einsichten der „Spielarten des Kapitalismus“-Schule in die institutionellen Voraussetzungen der Bildung spezifischen Humankapitals sei auch ein Schlüssel zum Verständnis der im internationalen Vergleich variierenden Grade an horizontaler und vertikaler Geschlechtersegregation gefunden.

Die Protagonisten der ,revisionistischen Wohlfahrtsstaatsdebatte“ stellen die Unsicherheit, die mit Investitionen in spezifisches Humankapital verbunden ist, in das Zentrum ihrer Argumentation (Abschn. 3.1). Diese Risiken indes, so Autoren wie Estévez-Abe (2006; siehe auch Estévez-Abe et al. 2001, S. 158-160), sind über die Geschlechter ungleich verteilt. Setzen hoch produktive Beschäftigungsverhältnisse Investitionen in spezifisches Humankapital voraus, werden Frauen die entsprechenden Investitionen scheuen, weil sie befürchten müssen, dass sich ihr Humankapital während Babypausen überdurchschnittlich entwertet. Ähnliches gilt für Arbeitgeber, die befürchten müssen, dass die betroffenen Beschäftigten den Arbeitsplatz zeitweilig verlassen und befristeter Ersatz nur schwer über den externen Arbeitsmarkt beschaffbar ist. Beides, so das Argument, senkt die Wahrscheinlichkeit, dass Investitionen weiblicher Beschäftigter in spezifisches Humankapital zustande kommen. Das aber bedeutet: Länder mit Institutionen, die in überdurchschnittlichem Maß auf die Formierung spezifischen Humankapitals hinwirken, werden mit hoher Wahrscheinlichkeit ein überdurchschnittliches Maß an geschlechtsspezifischer Segregation der Arbeitsmärkte aufweisen. Damit bietet das Argument eine theoretische Erklärung für den im internationalen Vergleich hoch signifikanten Zusammenhang zwischen dem Stellenwert beruflicher, im Unternehmen vollzogener Ausbildung und dem Frauenanteil im verarbeitenden Gewerbe (Estévez-Abe 2006, S. 164). ${ }^{14}$

Im selben argumentativen Fahrwasser zeigen verschiedene Autoren weitere, mitunter nicht minder überraschende Zusammenhänge zwischen Produktionsregime-Variablen einerseits und gender-bezogenen Ausprägungen andererseits. So argumentieren Iversen et al. (2005), es bestünde ein Kausalzusammenhang zwischen den Institutionen der Produktionsregime und Scheidungsraten: Je spezifischer das Humankapital hoch produktiver Arbeitsplätze, um so schlechter die Arbeitsmarktchancen nicht berufstätiger Frauen im Fall einer Ehescheidung und umso niedriger deshalb die tatsächlichen Scheidungsraten. Iversen u. Rosenbluth (2006) zeigen anhand von Umfragedaten, dass das Skill-Profil von Frauen (und damit, mittelbar, die institutionelle Ausgestaltung des Produktionsregimes) unter statistischer Kontrolle für weitere Variablen - die Arbeitsteilung innerhalb von Partnerschaften beeinflusst: Je höher die Arbeitsmarktchancen von Frauen, umso stärker sei

14 Das bedeutet im Umkehrschluss, dass die Geschlechtersegregation umso niedriger ausfällt, je mehr die berufliche Bildung von Schulen oder Universitäten erbracht wird: Je weiter weg vom Arbeitgeber, umso besser für den gleichberechtigten Berufszugang von Frauen (Estévez-Abe 2006, S. 153). 
ihre Position bei der Aushandlung der innerfamiliären Arbeitsteilung, und umso symmetrischer seien deshalb Tätigkeiten wie Putzen und Kochen innerhalb der Familien über die Geschlechter verteilt. ${ }^{15}$ Und Rosenbluth et al. (2002) sehen in derselben Argumentation einen potenziellen Beitrag zur Erklärung unterschiedlicher Fertilitätsraten entwickelter Industrieländer: Etwa seit den achtziger Jahren setzen hohe Geburtenraten ein hohes Maß an Vereinbarkeit von Familie und Beruf voraus (Castles 2003), und diese Vereinbarkeit sinkt mit dem Ausmaß an Spezifität des Humankapitals. ${ }^{16}$

\subsection{Makroökonomische Politik}

Auch das letzte Beispiel setzt am Problem der Humankapitalspezifität an, zielt aber auf Erklärung eines gänzlich anderen Sachverhalts: der Fähigkeit von Regierungen, mit ihrer Wirtschaftspolitik makroökonomische Aggregate zu steuern (Carlin u. Soskice 2009; Soskice 2006, 2007). Makroökonomische Politik, so die aus den Grundüberlegungen der „Spielarten des Kapitalismus“-Schule abgeleitete These, funktioniere in CMEs anders als in LMEs. Genauer: Die Reaktion der Binnennachfrage auf ökonomische Schocks ist abhängig von der Spezifität des Humankapitals. Geht das Wirtschaftswachstum zurück und nehmen die Beschäftigungsrisiken zu, so das Argument, werden die Mittelschichten in Ländern mit überdurchschnittlicher Spezifität des Humankapitals mit verstärkter Spartätigkeit, also mit einem makroökonomisch suboptimalen prozyklischen Nachfrageverhalten reagieren. Setzt in dieser Situation die Regierung auf fiskalische Expansion, werden die eingesetzten Mittel in überdurchschnittlichem Ausmaß in die Sparquote statt in den Konsum fließen. Die Multiplikatoreffekte, auf die antizyklische Fiskalpolitik zielt, werden nicht oder zumindest doch nur auf schwachem Niveau einsetzen. Im Ergebnis sinkt die Effektivität antizyklischer Fiskalpolitik. ${ }^{17}$

Hält dieser Grundgedanke, dann stellt die „Spielarten des Kapitalismus“-Schule ein Instrumentarium bereit, mit dem sich im alten Streit zwischen Neoklassikern und Keynesianern um die Frage, ob antizyklische Steuerungsversuche des Staats in Abschwungphasen sinnvoll sind, vermitteln lässt. Beide Schulen könnten gleichermaßen Recht und Unrecht haben, abhängig von den institutionellen Ausgestaltungen der Produktionsre-

15 Zwar können beschäftigungsintensive Wohlfahrtsstaaten, wie die Autoren zeigen, diesem Effekt gegensteuern. Das ändert allerdings nichts an dem exorbitanten Ausmaß an geschlechtsspezifischer Segregation der Arbeitsmärkte skandinavischer Länder. Die Autoren vermuten zudem, dass der gegensteuernde Effekt des Wohlfahrtsstaats auch erklärt, warum Frauen hinsichtlich ihrer politischen Präferenzen in den vergangenen ungefähr drei Jahrzehnten in nahezu allen entwickelten Industriestaaten spürbar nach links gewandert sind (und inzwischen ,linkere“ Präferenzen aufweisen als Männer): Die Interessen am Wohlfahrtsstaat sind über die Geschlechter ungleich verteilt (Iversen u. Rosenbluth 2006, siehe hier insbesondere die Ergebnisse in Regressionstabelle 3).

16 Auch hier gilt (wie bereits Castles 2003 herausstellte): Der Wohlfahrtsstaat kann, zumindest teilweise, für geschlechtsspezifische Benachteiligungen - hier: mangelnde Vereinbarkeit von Familie und Beruf - kompensieren.

17 Angemerkt sei an dieser Stelle, dass das skizzierte Argument lediglich ein Teil einer wesentlich umfassenderen Interpretation der Unterschiedlichkeit makroökonomischer Politik in CMEs und LMEs ist. 
gime, in denen die makroökonomische Politik ihre Wirkungen entfalten soll. Hatte die Politikwissenschaft in der Vergangenheit produktive Erkenntnisse über die Steuerungsfähigkeit der Regierungen angesichts ökonomischer Schocks hervorgebracht (grundlegend Scharpf 1987), ließe sich mit dem skizzierten Instrumentarium möglicherweise der Wissensstand über die im internationalen Vergleich variierende Steuerbarkeit makroökonomischer Aggregate erweitern.

Strengere empirische Tests dieser Erwartungen stehen noch aus. Jedoch scheint die Beobachtung international vergleichend operierender Ökonomen, der zufolge makroökonomische Steuerung in den achtziger und neunziger Jahren in Großbritannien und den USA besser gelang als in Japan und Deutschland (vergleiche etwa Heine et al. 2006), bemerkenswert gut zu Soskices Überlegungen zu passen. ${ }^{18}$ Möglicherweise könnte im selben Gedankengang auch eine potenzielle Erklärung für den Umstand liegen, dass im englischen Sprachraum seit längerem ein neo- bzw. postkeynesianischer Konsens im Entstehen begriffen scheint, nicht aber in Deutschland. Die Erfahrungen mit keynesianischer Makropolitik, so ließe sich als Hypothese formulieren, sind in den betroffenen Ländern unterschiedlich und übersetzen sich in Lehrmeinungen, die als allgemeine Theorien verabsolutiert werden, obwohl die doch eigentlich spezielle, von spezifischen Kontextfaktoren - den Institutionen der Produktionsregime - abhängige Theorien sein müssten.

\section{Fazit: „Spielarten des Kapitalismus“ als Schule der vergleichenden Staatstätigkeitsforschung}

In diesem Beitrag habe ich „Spielarten des Kapitalismus“ als Schule der vergleichenden Staatstätigkeitsforschung gewertet. Diese Wertung ist alles andere als selbstverständlich, ist VoC doch ursprünglich zur Aufdeckung anderer Zusammenhänge angetreten. Die institutionellen Grundlagen der Wettbewerbsfähigkeit von Unternehmen, die im Zentrum des Erkenntnisinteresses der Theorieschule stehen, scheinen auf den ersten Blick weit weg von typischen Problemen der Forschung über Staatstätigkeit. Tatsächlich aber, so habe ich gezeigt, haben sich die Elemente des Ansatzes - Unternehmenszentrierung, Unterscheidung von Koordinationsmodi und institutionellen Sphären, institutionelle Komplementarität, Unterscheidung unterschiedlicher Spielarten des Kapitalismus und Analyse der Implikationen für Wettbewerbskraft und institutionellen Wandel (Abschn. 2.1-2.6) - zu einer Perspektive verdichtet, aus der neuartige, nicht auf Kombinationen

18 Zudem wurde immer wieder gefragt, warum die Deutschen, anders als etwa die Amerikaner, insbesondere in schlechten Zeiten ein „Volk der Sparer“ sind. Als Antwort wurde auf die spezifisch deutschen ,historischen Erfahrungen“ verwiesen (so etwa FAZ vom 29.19.2008, S. 9), womit die Hyperinflation der frühen zwanziger Jahre sowie die Inflation nach dem Zweiten Weltkrieg gemeint sind. Diese historischen Erfahrungen mögen in der Tat eine überdurchschnittliche Inflationsabneigung erklären - aber eine hohe Sparneigung? Wären ausgeprägte Inflationsängste nicht eher eine Begründung für die (in Deutschland gerade nicht manifeste) Neigung, erworbenes Geld zügig auszugeben? Mir scheint, dass die produktionsregimebezogene Erklärung mögliche Antworten auf ein Puzzle bereithält, dessen traditionelle Lösung schon immer wenig überzeugend war. Freilich gilt auch hier, dass die entsprechenden Hypothesen empirischer Bestätigungen bedürfen, die noch ausstehen. 
der Annahmen anderer Schulen rückführbare Hypothesen zum Regierungshandeln generiert wurden. Fruchtbare Diskussionen, die hieraus entstanden sind, habe ich beispielhaft anhand der Analyse wohlfahrtsstaatlicher Politik, der Geschlechtersegregation und der makroökonomischen Politik nachgezeichnet (Abschn. 3.1-3.3). Im Ergebnis schlage ich vor, die Unterscheidung von Schulen der vergleichenden Staatstätigkeitsforschung wie folgt zu erweitern: ${ }^{19}$

1. Theorien der sozioökonomischen Determination

2. Machtressourcen-Theorien

3. Theorien der Parteiendifferenz

4. Politisch-institutionalistische Theorien

5. Theorien der Internationalisierung und der De-Industrialisierung

6. Spielarten des Kapitalismus

Was bedeutet diese Erweiterung für die vergleichende Staatstätigkeitsforschung? Sie leitet zum einen zur Berücksichtigung von VoC-spezifischen Hypothesen im konkreten Forschungsprozess an. Bestätigen sich die aus VoC abgeleiteten Hypothesen im qualitativen oder quantitativen Test, kann der spezifische Erklärungswert der Theorieschule additiv zu komplementären Befunden interpretiert werden. So mag die Berücksichtigung von VoC beispielsweise bei der Analyse von Sozialquoten den Anteil erklärter Varianz an der Gesamtvarianz erhöhen, ohne dass dies direkte Konsequenzen für die Befunde zur Erklärungskraft politisch-institutioneller oder parteipolitischer Variablen nach sich zöge. Denkbar ist aber auch, dass die Aufnahme von VoC-Hypothesen in das Analyseraster zur Verkleinerung des Erklärungswerts konkurrierender Hypothesen führt. So mögen Anteile erklärter Varianz, die früher der Erklärungskraft von Klassentheorien zugeschrieben wurden, nunmehr auf die Erklärungskraft der Humankapitalproduktions-Theorie entfallen, ohne dass sich der Anteil erklärter Varianz an der Gesamtvarianz erhöhen würde. Im Ergebnis würde die Berücksichtigung der „Spielarten des Kapitalismus“-Schule die Erklärungskraft konkurrierender Schulen einem härteren empirischen Test aussetzen. ${ }^{20}$ Diese Überlegungen gelten unabhängig davon, ob mit quantitativ-ländervergleichenden Tests, mit qualitativen Vergleichen kleiner Fallzahl oder mit Fallstudien operiert wird.

Die Implikationen greifen aber tiefer. Die im oben beschriebenen Sinne additive Behandlung der neu gewonnen Befunde ändern nichts daran, dass - technisch gesprochendas Ziel der vergleichenden Analyse in der Schätzung von über Länder- und Sequenzgrenzen hinweg konstanten Regressionskoeffizienten und deren Zuordnung zu konkurrie-

19 Vergleiche Fußnote 1 zur These einer theoriegeschichtlichen Abfolge in Schmidts Vorschlag der Unterscheidung von Schulen der vergleichenden Staatstätigkeitsforschung.

20 In diesem Text habe ich die Unterscheidung von Schulen der Staatstätigkeitsforschung zum Ausgangspunkt genommen und für eine Erweiterung um VoC plädiert. Daraus folgt ein paralleles Argument, das gegenüber Vertretern der VoC-Schule zu führen wäre: Produktiv nutzbar ist VoC vor allem, wenn man sich seine Stellung als eine unter mehreren komplementären Schulen vor Augen führt und das Instrumentarium bei Bedarf mit den Instrumentarien anderer Schulen koppelt. Im Kontext der VoC-Diskussion versteht sich diese Aussage alles andere als von selbst. Vergleiche etwa Hancké et al. (2007b), die für den Ausbau von VoC zu einer "Generaltheorie" zu plädieren scheinen, die Antworten auf alle nur denkbaren Probleme der vergleichenden Politischen Ökonomie bereithalten soll. 
renden, möglicherweise komplementären Erklärungsansätzen besteht. ${ }^{21}$ „Spielarten des Kapitalismus“" als Schule der vergleichenden Staatstätigkeitsforschung ernst zu nehmen, impliziert aber, der Möglichkeit Rechnung zu tragen, dass die in den Koeffizienten zum Ausdruck kommende Konstanz aufzudeckender Kausalität in der Realität nicht existiert. Wenn die Funktionalität beispielsweise des Kündigungsschutzes systematisch zwischen unterschiedlichen Spielarten des Kapitalismus variiert, warum sollen dann die hinter der Institution stehenden politischen Dynamiken, nach deren Offenlegung die vergleichende Staatstätigkeitsforschung strebt, über Ländergrenzen hinweg konstant sein?

In diesem Sinne leitet VoC zu einer grundsätzlicheren Überprüfung von Grundprämissen der Staatstätigkeitsforschung an, die beispielsweise dadurch erfolgen kann, dass Kausalitäten zunächst ländergruppenspezifisch zugeschrieben werden und die Verallgemeinerung über Länder- oder Ländergruppengrenzen hinweg erst dann erfolgt, wenn sich erweist, dass sich die aufgedeckten Kausalitäten nicht oder nur in begrenztem Umfang unterscheiden. Da aber die quantitative Forschung wegen der begrenzten Anzahl der auf unterschiedliche Spielarten des Kapitalismus entfallenden Länder - etwa die bei Amable unterschiedenen fünf Ländergruppen (siehe Abschn. 2.5) - und der damit einhergehenden Reduktion statistischer Freiheitsgrade an Grenzen stoßen würde, spricht die Wertung von „Spielarten des Kapitalismus“ als Schule der vergleichenden Staatstätigkeitsforschung für eine vermehrte Kopplung qualitativer und quantitativer Forschungsstrategien. Möglicherweise trägt $\mathrm{VoC}$ in diesem Sinne dazu bei, dass sich Verfechter beider Forschungsstrategien, die sich in der Forschungspraxis häufig leider allzu wenig zu sagen haben, in der Staatstätigkeitsforschung künftig vermehrt aufeinander beziehen.

Open Access: Dieser Artikel unterliegt den Bedingungen der Creative Commons Attribution Noncommercial License. Dadurch sind die nichtkommerzielle Nutzung, Verteilung und Reproduktion erlaubt, sofern der/die Originalautor/en und die Quelle angegeben sind.

\section{Literatur}

Aglietta, Michel. 1976. A theory of capitalist regulation: The US experience. London: Verso. Amable, Bruno. 2003. The diversity of modern capitalism. Oxford: Oxford University Press. Amable, Bruno, und Pascal Petit. 2001. The diversity of social systems of innovation and production during the 1990s. Paris: Centre d'Etudes Prospectives d'Economie Mathematique Appliquees a la Planification.

Amable, Bruno, und Stefano Palombarini. 2009. A neorealist approach to institutional change and the diversity of capitalism. Socio-Economic Review 7:123-43.

Boyer, Robert. 1990. The regulation school: A critical introduction. Translated by Craig Charney. New York: Columbia University Press.

21 Das selbe gilt für das in den neunziger Jahren entwickelte "Families of Nations"-Konzept (siehe die Beiträge in Castles 1993). Zwar wurden hier, ähnlich wie bei der „Spielarten“-Schule (siehe Abschn. 2.5), Länder zu politökonomisch eng verwandten Ländergruppen zusammengefasst. Aber die kausalen Mechanismen, die die zwischen Ländern variierenden Politikergebnisse hervorbrachten, waren raum- und zeitunabhängige Konstanten, keine Variablen. 
Boyer, Robert. 1998. Hybridization and models of production: Geography, history, and theory. In The transfer and hybridization of productive models in the international automobile industry between imitation and inovation, eds. Robert Boyer et al., 23-56. Oxford: Oxford University Press.

Boyer, Robert. 2000. Is a finance led growth regime a viable alternative to fordism? A preliminary analysis. Economy \& Society 29:111-45.

Boyer, Robert, und Yves Saillard. 2002. Regulation theory: The state of the art. Translated by Carolyn Shread. London: Routledge.

Bruno, Michael, und Jeffrey D. Sachs. 1985. Economics of worldwide stagflation. Cambridge: Havard University Press.

Busch, Andreas. 1995. Preisstabilitätspolitik: Politik und Inflationsraten im internationalen Vergleich. Opladen: Leske und Budrich.

Calmfors, Lars, und John Driffill. 1988. Bargaining structure, corporatism and macroeconomic performance. Economic Policy 3:13-61.

Carlin, Wendy, und David Soskice. 2009. German economic performance: Disentangling the role of supply-side reforms, macroeconomic policy and coordinated economy institutions. SocioEconomic Review 7:67-99.

Castles, Francis G. 1993, Hrsg. Families of nations: Patterns of public policy in western democracies. Brookfield: Dartmouth.

Castles, Francis. G. 2003. The world turned upside down: Below replacement fertility, changing preferences and family-friendly public policy in 21 OECD countries. Journal of European Social Policy 13:209-27.

Crepaz, Markus L. 1992. Corporatism in decline? An empirical analysis of the impact of corporatism on macroeconomic performance and industrial disputes in 18 industrialized democracies. Comparative Political Studies 25:139-68.

Crouch, Colin. 1990. Trade unions in the exposed sector: Their influence on neo-corporatist behaviour. In Labor relations and economic performance, eds. Renato Brunetta, und Carlo Dell'Aringa, 68-91. London: Macmillan.

Czada, Roland. 1983. Konsensbedingungen und Auswirkungen neokorporatistischer Politikentwicklung. Journal für Sozialforschung 23:421-39.

Dell' Aringa, Carlo, und Manuela Samek Lodovici. 1992. Industrial relations and economic performance.: In participation in public policy making. The role of trade unions and employers' associations, ed. Tiziano Treu, 26-58. Berlin: de Gruyter.

Esping-Andersen, Gøsta. 1990. The three worlds of welfare capitalism. Oxford: Polity Press.

Estévez-Abe, Margarita. 2006. Gendering the varieties of capitalism: A study of occupational segregation by sex in advanced industrial societies. World Politics 59:142-75.

Estévez-Abe, Margarita, Torben Iversen, und David Soskice. 2001. Social protection and the formation of skills: A reinterpretation of the welfare state. In Varieties of capitalism. the institutional foundations of comparative advantage, eds. Peter A. Hall, und David Soskice, 145-83. Oxford: Oxford University Press.

Freeman, Richard B. 1988. Labour market institutions and economic performance. Economic Policy 6:63-80.

Hall, Peter A. 2005. Institutional complementarity: Causes and effects. Socio-Economic Review 3:373-77.

Hall, Peter A. 2007. The evolution of varieties of capitalism in Europe. In Beyond varieties of capitalism: Conflict, contradictions, and complementarities in the European economy, eds. Bob Hancké, Martin Rhodes, und Mark Thatcher, 29-85. New York: Oxford University Press.

Hall, Peter A., und Daniel W. Gingerich. 2004. Varieties of capitalism and institutional complementarities in the political economy: An empirical analysis. MPIfG Discussion Paper 2004/5. Köln: Max Planck Institute for the Study of Societies. 
Hall, Peter A., und David Soskice. 2001a. An introduction to varieties of capitalism. In Varieties of capitalism: Institutional foundations of comparative advantage, eds. Peter A. Hall, und David Soskice, 1-68. Oxford: Oxford University Press.

Hall, Peter A., und David Soskice, eds. 2001b. Varieties of capitalism: The institutional foundations of comparative advantage. Oxford: Oxford University Press.

Hall, Peter A., und Kathleen Thelen. 2009. Institutional change in varieties of capitalism. SocioEconomic Review 7:7-34.

Hancké, Bob, Martin Rhodes, und Mark Thatcher, eds. 2007a. Beyond varieties of capitalism: Conflict, contradictions, and complementarities in the European economy. Oxford: Oxford University Press.

Hancké, Bob, Martin Rhodes, und Mark Thatcher. 2007b. Introduction: Beyond varieties of capitalism. In Beyond varieties of capitalism: Conflict, contradictions, and complementarities in the European economy, eds. Bob Hancké, Martin Rhodes, und Mark Thatcher, 3-38. New York: Oxford University Press.

Heine, Michael, Hansjörg Herr, und Cornelia Kaiser. 2006. Wirtschaftspolitische Regime westlicher Industrienationen. Baden-Baden: Nomos.

Heitger, Bernhard. 1987. Corporatism, technological gaps and growth in OECD countries. Weltwirtschaftliches Archiv 123:463-73.

Hibbs, Douglas. 1977. Political parties and macroeconomic policies. American Political Science Review 71:467-87.

Hibbs, Douglas. 1992. Partisan theory after fifteen years. European Journal of Political Economy 8:361-76.

Hicks, Alexander, und Lane Kenworthy. 1998. Cooperation and political economic performance in affluent democratic capitalism. American Journal of Sociology 103:1631-72.

Höpner, Martin. 2005. What have we learnt? Complementarity, coherence and institutional change. Socio-Economic Review 3:383-7.

Höpner, Martin. 2007. Coordination and organization: The two dimensions of nonliberal capitalism. MPIfG Discussion Paper 2007/12. Köln: Max-Planck-Institut für Gesellschaftsforschung.

Höpner, Martin, Alexander Petring, Daniel Seikel, und Benjamin Werner. 2009. Liberalisierungspolitik. Eine Bestandsaufnahme von zweieinhalb Dekaden marktschaffender Politik in entwickelten Industrieländern. MPIfG Discussion Paper 2009/7. Köln: Max-Planck-Institut für Gesellschaftsforschung.

Howell, Chris. 2003. Varieties of capitalism: And then there was one? Comparative Politics 36:103-24.

Iversen, Torben. 2005. Capitalism, democracy and welfare. Cambridge: Cambridge University Press.

Iversen, Torben. 2006. Responses and some agenda items for the future study of democratic capitalism. Labor History 47:439-49.

Iversen, Torben, und David Soskice. 2001. An asset theory of social policy preferences. American Political Science Review 95:875-93.

Iversen, Torben, und Frances Rosenbluth. 2006. The political economy of gender: Explaining crossnational variation in the gender division of labor and the gender voting gap. American Journal of Political Science 50:1-19.

Iversen, Torben, und John D. Stephens. 2008. Partisan politics, the welfare state, and three worlds of human capital formation. Comparative Political Studies 4:600-37.

Iversen, Torben, und Thomas Cusack. 2000. The causes of welfare state expansion: Deindustrialization or globalization? World Politics 52:313-49.

Iversen, Torben, Frances Rosenbluth, und David Soskice. 2005. Divorce and the gender division of labor in comparative perspective. Social Politics 12:216-42.

Keynes, John Maynard. 1926. Das Ende des Laissez-Faire. Ideen zur Verbindung von Privat- und Gemeinwirtschaft. München: Duncker \& Humblot. 
Kitschelt, Herbert. 2006. Collective group interests and distributive outcomes: Competing claims about the evolution of the welfare state. Labor History 47:397-449.

Korpi, Walter. 1978. The working class in welfare capitalism: Work, unions and politics in Sweden. London: Routledge.

Korpi, Walter. 2006. Power resources and employer-centered approaches in explanations of welfare states and varieties of capitalism: Protagonists, consenters, and antagonists. World Politics 58:167-206.

Lijphart, Arend. 1999. Patterns of democracy: Government forms and performance in thirty-six countries. New Haven: Yale University Press.

Mares, Isabela. 2000. Strategic alliances and social policy reform: Unemployment insurance in comparative perspective. Politics \& Society 28:223-44.

Mares, Isabela. 2001. Firms and the welfare state: When, why, and how does social policy matter to employers? In Varieties of capitalism: The institutional foundations of comparative advantage, eds. Peter A. Hall, und David Soskice, 184-212. Oxford: Oxford University Press.

Mares, Isabela. 2003. The politics of social risk: Business and welfare state development. Cambridge: Cambridge University Press.

Marx, Karl, und Friedrich Engels. 1848/1973. Manifest der Kommunistischen Partei. Berlin: Dietz.

Mayntz, Renate, und Fritz W. Scharpf. 1995. Steuerung und Selbstorganisation in staatsnahen Sektoren. In Gesellschaftliche Selbstregulierung und politische Steuerung, Hrsg. Renate Mayntz, und Fritz W. Scharpf, 9-38. Frankfurt: Campus.

Meltzer, Allan H., und Scott F. Richard. 1981. A rational theory of the size of government. Journal of Political Economy 89:914-27.

North, Douglass C., und Robert Paul Thomas. 1973. The rise of the western world: A new economic history. Cambridge: Cambridge University Press.

Obinger, Herbert, Uwe Wagschal, und Bernhard Kittel, Hrsg. 2003. Politische Ökonomie. Demokratie und wirtschaftliche Leistungsfähigkeit. Wiesbaden: VS Verlag.

Olson, Mancur. 1982. The rise and decline of nations: Economic growth, stagflation, and social rigidities. New Haven: Yale University Press.

Pierson, Paul. 2000. Three worlds of welfare state research. Comparative Political Studies 33:791-821.

Rosenbluth, Frances, Matthew Light, und Claudia Schrag. 2002. The politics of low fertility: Global markets, women's employment, and birth rates in four industrialized democracies (unpubliziertes Manuskript).

Scarpetta, Stefano. 1996. Assessing the role of labour market policies and institutional settings on unemployment: A cross-country study. OECD Economic Studies 26:43-98.

Scharpf, Fritz W. 1985. Plädoyer für einen aufgeklärten Institutionalismus. In Policy-Forschung in der Bundesrepublik Deutschland. Ihr Selbstverständnis und ihr Verhältnis zu den Grundfragen der Politikwissenschaft, Hrsg. Hans-Hermann Hartwich, 164-70, Opladen.

Scharpf, Fritz W. 1987. Sozialdemokratische Krisenpolitik in Europa. Frankfurt: Campus.

Scharpf, Fritz W. 1999. Regieren in Europa: Effektiv und demokratisch? Frankfurt: Campus.

Schmid, Josef. 1993. Parteien und Verbände. Konstitution, Kontingenz und Koevolution im System der Interessenvermittlung. In Verhandlungsdemokratie, Interessenvermittlung, Regierbarkeit. Festschrift für Gerhard Lehmbruch, eds. Roland Czada, und Manfred G. Schmidt, 171-89. Opladen: Westdeutscher Verlag.

Schmidt, Manfred G. 1982. Wohlfahrtsstaatliche Politik unter bürgerlichen und sozialdemokratischen Regierungen. Ein internationaler Vergleich. Frankfurt: Campus.

Schmidt, Manfred G. 1995. Vergleichende Politikforschung mit Aggregatdaten: Inwieweit beeinflussen Parteien Regierungspolitik? In Politikwissenschaftliche Methoden. Grundriss für Studien und Forschung. Hrsg. Ulrich von Alemann, 327-56. Opladen: Westdeutscher Verlag. 
Schmidt, Manfred G. 2002. The impact of political parties, constitutional structures and veto players on public policy. In Comparative democratic politics: A guide to contemporary theory and research, ed. Hans Keman, 166-84. London: Sage.

Schmidt, Manfred G. 2008. Demokratietheorien. Eine Einführung. 4., überarbeitete und erweiterte Auflage. Wiesbaden: VS Verlag.

Schmidt, Manfred G., Tobias Ostheim, Nico A. Siegel, und Reimut Zohlnhöfer, Hrsg. 2007. Der Wohlfahrtsstaat: Eine Einführung in den historischen und internationalen Vergleich. Wiesbaden: VS Verlag.

Schmidt, Vivien A. 2002. The futures of European capitalism. Oxford: Oxford University Press.

Schmidt, Vivien A. 2008. European political economy: Labour out, state back in, firm to the fore. west european politics. European Political Economy 31:302-20.

Schumpeter, Joseph A. 1942/1950. Kapitalismus, Sozialismus und Demokratie. Einleitung von Edgar Salin. Bern: Francke Verlag.

Soskice, David. 1990. Wage Determination: The changing role of institutions in advanced industrialized countries. Oxford Review of Economic Policy 6:36-61.

Soskice, David. 1999. Divergent production regimes: Coordinated and uncoordinated market economies in the 1980s and 1990s. In Continuity and change in contemporary capitalism, eds. Herbert Kitschelt, Peter Lange, Gary Marks, und John D. Stephens, 101-34. Cambridge: Cambridge University Press.

Soskice, David. 2006. Skill specificity and the modern macroeconomics of unemployment: Using the iversen tool-kit. Labor History 47:429-39.

Soskice, David. 2007. Macroeconomics and varieties of capitalism. In Beyond varieties of capitalism: Conflict, contradictions, and complementarities in the european economy, eds. Bob Hancké, Martin Rhodes, und Mark Thatcher, 89-121. New York: Oxford University Press.

Stephens, John D. 2006. Partisan government, employers' interests, and the welfare state. Labor History 47:420-29.

Streeck, Wolfgang. 2005. Requirements for a useful concept of complementarity. Socio-Economic Review 3:363-6.

Streeck, Wolfgang. 2009. Re-forming capitalism: Institutional change in the German political economy. New York: Oxford University Press.

Streeck, Wolfgang, und Philippe C. Schmitter. 1985. Gemeinschaft, Markt und Staat - und die Verbände? Der mögliche Beitrag von Interessenregierungen zur sozialen Ordnung. Journal für Sozialforschung 25:133-57.

Swenson, Peter. 1991. Bringing capital back in, or social democracy reconsidered: Employer power, cross-class alliances, and centralization of industrial relations in Denmark and Sweden. World Politics 43:513-44.

Swenson, Peter A. 2002. Capitalists against markets: The making of labor markets and welfare states in the United States and Sweden. Oxford: Oxford University Press.

Swenson, Peter A. 2004. Varieties of capitalist interests: Power, institutions, and the regulatory welfare state in the United States and Sweden. Studies in American Political Development 18:1-29.

Taylor, Mark Zachary. 2004. Empirical evidence against varieties of capitalism's theory of technological innovation. International Organization 58:601-31.

Wagner, Adolph. 1911/1970. Das Gesetz der zunehmenden Staatstätigkeit. In Finanztheorie, Hrsg. Horst Claus Recktenwald, 241-7. Köln: Kiepenheuer \& Witsch.

Wagschal, Uwe, und Markus Freitag. 2007. Direkte Demokratie - Bestandsaufnahmen und Wirkungen im internationalen Vergleich. Münster: Lit Verlag.

Wilensky, Harold L., und Charles N. Lebeaux. 1958. Industrial society and social welfare. New York: Free Press.

Zohlnhöfer, Reimut. 2005. Globalisierung der Wirtschaft und nationalstaatliche Anpassungsreaktionen. Theoretische Überlegungen. Zeitschrift für Internationale Beziehungen 12:41-75. 
Zohlnhöfer, Reimut. 2007. Stand und Perspektiven der vergleichenden Staatstätigkeitsforschung. In Die Zukunft der Policy-Forschung: Theorien, Methoden, Anwendungen, Hrsg. Frank Janning, und Katrin Toens. Wiesbaden: VS Verlag. 